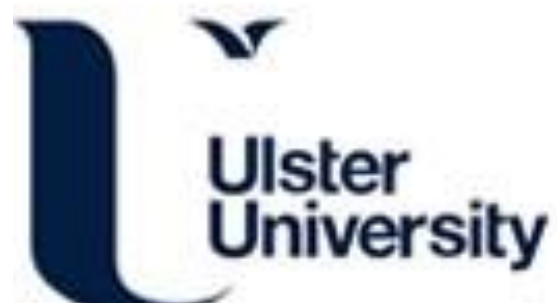

\section{Towards increasing the number of commands in a hybrid brain computer-interface with combination of gaze and motor imagery}

Meena, Y., Cecotti, H., Wong-Lin, K., \& Prasad, G. (2015). Towards increasing the number of commands in a hybrid brain computer-interface with combination of gaze and motor imagery. In Unknown Host Publication IEEE.

Link to publication record in Ulster University Research Portal

\section{Published in:}

Unknown Host Publication

Publication Status:

Published (in print/issue): 29/08/2015

\section{Document Version}

Publisher's PDF, also known as Version of record

\section{General rights}

Copyright for the publications made accessible via Ulster University's Research Portal is retained by the author(s) and / or other copyright owners and it is a condition of accessing these publications that users recognise and abide by the legal requirements associated with these rights.

\section{Take down policy}

The Research Portal is Ulster University's institutional repository that provides access to Ulster's research outputs. Every effort has been made to ensure that content in the Research Portal does not infringe any person's rights, or applicable UK laws. If you discover content in the Research Portal that you believe breaches copyright or violates any law, please contact pure-support@ulster.ac.uk. 


\title{
Towards increasing the number of commands in a hybrid brain- computer interface with combination of gaze and motor imagery
}

\author{
Yogesh Kumar Meena, Student Member, IEEE, Hubert Cecotti, Member, IEEE, KongFatt Wong-Lin, \\ Member, IEEE., and Girijesh Prasad, Senior Member, IEEE
}

\begin{abstract}
Non-invasive brain-computer interface (BCI) provides a novel means of communication. This can be achieved by measuring electroencephalogram (EEG) signal over the sensory motor cortex of a person performing motor imagery (MI) tasks. However, the performance of BCI remains currently too low to be of wide practical use. A hybrid BCI system could improve the performance by combining two or more modalities such as eye tracking, and the detection of brain activity responses. In this paper, first, we propose a simultaneous hybrid BCI that combines an event-related desynchronization (ERD) BCI and an eye tracker. Second, we aim to further improve performance by increasing the number of commands (i.e., the number of choices accessible to the user). In particular, we show a significant improvement in performance for a simultaneous gaze-MI system using a total of eight commands. The experimental task requires subjects to search for spatially located items using gaze, and select an item using MI signals. This experimental task studied visuomotor compatible and incompatible conditions. As incorporating incompatible conditions between gaze direction and MI can increase the number of choices in the hybrid BCI, our experimental task includes single-trial detection for average, compatible and incompatible conditions, using seven different classification methods. The mean accuracy for MI, and the information transfer rate (ITR) for the compatible condition is found to be higher than the average and the incompatible conditions. The results suggest that gaze-MI hybrid BCI systems can increase the number of commands, and the location of the items should be taken into account for designing the system.
\end{abstract}

\section{INTRODUCTION}

Non-invasive brain-computer interface $(\mathrm{BCI})$ provides a communication pathway for healthy as well as differentlyabled people such as those with severe motor impairments. With the aid of analyzed EEG signals, BCI systems have the potential to be used in neuro-rehabilitation, controlling robots, virtual keyboard and other augmentative devices, and entertainment such as video games, switching control, and virtual automobile control [1],[2]. Current BCIs are mainly dependent on the cognitive functions associated with motor imagery (MI) [3]. A BCI system can be devised by measuring EEG signal over sensory-motor cortex, while a user is performing an MI task [4]. The vividness of MI is highly dependent on visual perception and imagery. However, current BCI systems' performance remains too low to allow users to fully exploit the advantages [5]. Although

Y.K.M. is supported by the Government of India (Education11016152013). G.P., K.W.-L., and H.C. are supported by the Northern Ireland Functional Brain Mapping Facility (1303/101154803), funded by InvestNI, University of Ulster, Magee Campus, Northland Road, BT48 7JL, Northern Ireland (phone: +44 28 716 75740; e-mail: Yogesh Kumar, Meena <meena-y@email.ulster.ac.uk>). some research groups have claimed to achieve systems' accuracy of up to $96 \%$ [6],[7] they came along with limitations such as low information transfer rate (ITR), reliability, and user acceptability. By incorporating other modalities, i.e. hybrid BCIs, the performance can be improved.

To design a hybrid BCI system for clinical and rehabilitation purposes, the constraints of the patients have to be considered. For example, for patients who are not completely locked-in (e.g., quadriplegic), they may still be able to control their gaze and a hybrid BCI can exploit this ability [8],[9]. The recording of gaze position may be done using an eye-tracker system, which measures eye movements and positions with two major monitoring features. The first feature measures the position of the eye relative to the head, and the second feature measures the orientation of the eye in space (point of regard). The recorded eye position and movement data can be analyzed to determine the pattern, duration of eye fixations (dwell time), and the sequence of the scan paths on a screen [10],[11].

An eye-tracker can be used independently to search and select an item; the searching is done by gaze coordinates and the item selection may be done by the dwell time [3],[12]. Therefore, the dwell time should be sufficiently long enough for a correct selection of the intended item. Otherwise, high false selections may result, leading to high level of frustration in the user and thus delaying the overall process. A hybrid BCI may overcome such dwell time issue, by a combination of two or more different systems, often combining different neurophysiological (e.g., EEG) signals, or neurophysiological with other physiological input signal sources [13],[8]. For designing hybrid BCIs, the systems can be combined sequentially or simultaneously [13],[8],[14]. Furthermore, to enhance the effectiveness and use of hybrid BCIs, it is important to increase the number of commands for the user. This can be achieved through combining the gaze and the brain responses (i.e., MI, SSVEP, and P300) [8], [9],[15],[16].

In this work, we propose a novel simultaneous gaze-MI hybrid BCI (see Fig. 1) to increase the number of commands from two or four to eight. The system is based on the item search through gaze, and item selection by the EEG response detection during an MI task simultaneously on the computer screen. The novelty of the task stems from the inclusion of visuomotor compatible and incompatible task conditions to allow more potential choices to the user. However, as far as we know, there is no study in BCI that evaluates the performance of incompatible task conditions. In our incompatible condition, a word "Left" would appear on the right of the screen, or a word "Right" appearing on the left of 


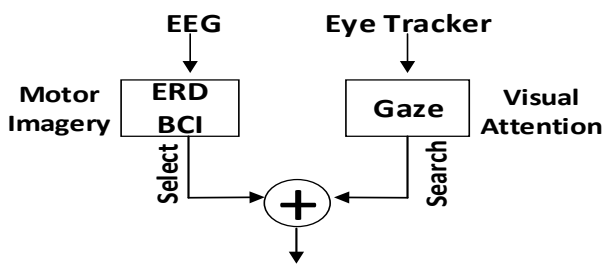

Fig 1: A simultaneous hybrid BCI that combines an ERD BCI and an eye tracker, wherein an item is searched through gaze and the item selection is made by motor imagery simultaneously.

the screen. Several signal processing techniques are tested on these data. Our results show that the accuracy for MI and the ITR of the compatible condition is higher than that of the average and the incompatible condition. This paper is organized as follows: Section II discusses the experimental protocol. Section III is dedicated to the methods used. Section IV presents the results. Finally, Section V provides a discussion and conclusion.

\section{EXPERIMENTAL PROTOCOL}

\section{A. Subjects}

Seven consenting healthy male subjects participated in the study. They were in the age range 21-35 years, (mean age of 28.7 and standard deviation=4.2). All subjects had no prior experience with an eye tracker. None of the subjects had any visual or neurological conditions. Prior to experiments, every subject was advised about the nature and purpose of the study. No financial reward was received by the subjects for their participation in the study. The experiments were conducted with full consent of the subjects as per the revised Helsinki Declaration of 2000.

\section{B. Design and Operational Procedure}

Fig. 1 depicts the proposed model of simultaneous hybrid BCI system. The experiment consisted of a search and select task in a two dimensional environment. It is mainly divided into two categories. The recording is done simultaneously for both EEG and eye movement data from subjects. The eye tracker is used as a searching device, which has a direct mapping to the mouse cursor, providing a real-time feedback response. The BCI was used to provide an additional selection command to the user, using left or right hand MI. We have used a visual cue paradigm for stimulus representation on a computer screen. It consists of four visual stimuli, each containing a fixation cross to fixate the gaze. Two visual stimuli are located on each side of the screen, with an additional visual stimulus located at the center of the screen, representing an idle state (Fig. 2 (A)-(B)).

Subjects used the eye tracker controlled search command to direct the cursor to the target visual stimuli, whilst attempting the prompted select command by left or right hand MI. A rigid and stable eye tracker head lock was used for positioning the subjects' head at the center of the screen. Each subject was seated in a comfortable chair located in front of the eye tracker head lock and approximately $55 \mathrm{~cm}$ from a 22 inch LCD monitor. Lighting conditions were maintained constant during the experiments.
(A)

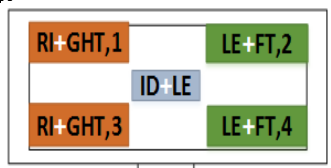

(B)
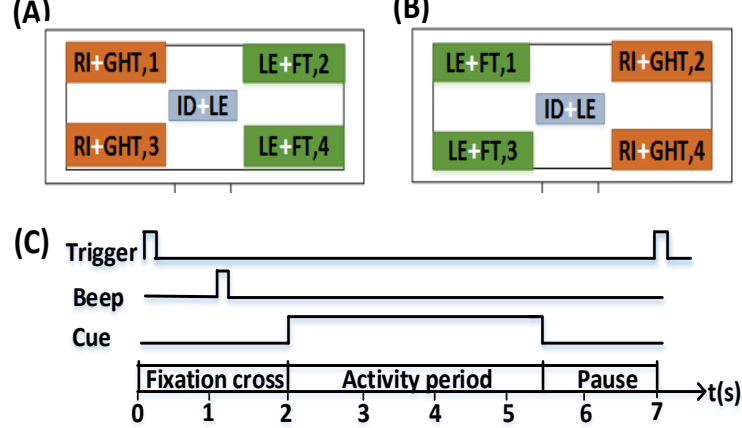

Fig 2: Proposed hybrid BCI and timing scheme of the paradigm. Computer screen in (A) represents an incompatible state of imagining right and left hand movements with four gaze coordinates $(1,2,3,4)$, Computer screen in (B) shows a compatible state of imagination of right and left hand movements with four gaze coordinates $(1,2,3,4)$. (C) Timing scheme of the single trial paradigm.

Prior to an experiment, the eye tracker was calibrated using a 16-point calibration scheme. This provides each subject with an accurate position of his/her gaze. Each experiment consists of one session, comprising of 40 trials each lasting about $7 \mathrm{~s}$. In each trial, the target of interest appears in a pseudo-random order with equal probability but they are evenly distributed over a session, appearing 10 times each. During each trial, subjects were prompted to search for a single target of interest on the visual display using the eye tracker controlled mouse cursor, while attempting a left or right hand MI command. Fig. 2 (C) shows a timing scheme of the paradigm. A beep command is issued at $1 \mathrm{~s}$ to indicate a get ready signal, with a visual cue appearing at $2 \mathrm{~s}$. The visual cue, a colored rectangle with a fixation cross, appears for a period of $3.5 \mathrm{~s}$, and during this period subjects would perform the search and select assessment task. On/off-set times should not be deterministic to minimize anticipatory responses. Each session lasts about 5 minutes. For each subject, one session is provided.

\section{Data Acquisition}

The eye tracker signals were recorded from an Arrington Research Eye Tracker system comprising of a monocular high resolution infrared camera, which records gaze $\mathrm{x}$, gaze $\mathrm{y}$, trigger, and label indexes at $128 \mathrm{~Hz}$ sampling rate. The EEG signal was recorded at a $128 \mathrm{~Hz}$ sampling rate using the gUSBamp and g.SAHARA dry electrode system from g.Tec. It consisted of two bipolar recordings ( $\mathrm{C} 3$ and $\mathrm{C} 4)$, with the right mastoid serving as ground. A bandpass-filter between $0.5 \mathrm{~Hz}$ and $60 \mathrm{~Hz}$, and a notch filter at $50 \mathrm{~Hz}$ were enabled.

\section{METHODS}

\section{A. Gaze Detection}

Eye tracker signals i.e., gaze $\mathrm{x}$ - and $\mathrm{y}$-coordinates were distributed according to label indexes (1, 2, 3, and 4). The label indexes were used to identify one of the four classes and these indexes show the start activity in each trial. A median filter was applied to the eye tracker data to remove eye blinks and the mean gaze position in horizontal and vertical directions were recorded for each trial. The distance of $\mathrm{x}$ - and $\mathrm{y}$-coordinates from each visual stimulus was calculated, and corresponding to the minimum distance from 
the gaze coordinates, the actual output was estimated according to label indexing. The confusion matrix was used to compute the accuracy.

\section{B. Feature Extraction and classification}

Band-power features for all the channels are extracted for $\mathrm{mu}(\mu)(8-12 \mathrm{~Hz})$ and beta $(\beta)(12-30 \mathrm{~Hz})$ rhythms. During each trial, the time over the imagery period is selected for extracting the band-power features. This information called event-related de-synchronization (ERD) and event-related synchronization (ERS) over $\mu$ and $\beta$ frequency bands are used for classification. The ERD/ERS can be seen as a correlate of an activated cortical area, so as to effectively account for ERD and ERS phenomena observed during MI tasks. The performance of the proposed hybrid system was assessed by seven binary classifiers: support vector machines (SVM) with linear kernel, quadratic kernel, q-polynomial kernel, Gaussian kernel, k-nearest neighbors (kNN) with $(\mathrm{K}=1$ and $\mathrm{K}=5)$, and linear discriminant analysis (LDA). The performance evaluation was performed with a ten-fold cross validation. In the next section, we report the accuracy (in \%) and the ITR [9],[17] in bit per symbol (bps).

\section{RESULTS}

\section{A. Gaze Detection}

The performance evaluation of gaze detection was based on 40 trials per subject. The minimum distance between the fixation point (i.e. the center), and the detected gaze location was used to assign the item selected by the user. The offline gaze detection (eye tracker only) results are presented in Table I. The mean accuracy for all subjects is $95.2 \pm 6.3 \%$.

\section{B. Single-Trial Detection}

To evaluate the performance of single-trial detection we considered two classes - left and right hand MI, with respect to the four events - left computer screen $(1,3)$ and right computer screen $(2,4)$. The single-trial detection results were computed in the form of visuomotor compatible and incompatible conditions for each subject. Accordingly, the single-trial detection results are divided into three classes: $\left(\mathrm{L}_{\mathrm{M}}\right.$ vs $\left.\mathrm{R}_{\mathrm{M}}\right) ;\left(\mathrm{L}_{\mathrm{M}} \cap \mathrm{L}_{\mathrm{S}}\right)$ vs $\left(\mathrm{R}_{\mathrm{M}} \cap \mathrm{R}_{\mathrm{S}}\right) ;\left(\mathrm{L}_{\mathrm{M}} \cap \mathrm{R}_{\mathrm{S}}\right)$ vs $\left(\mathrm{R}_{\mathrm{M}} \cap\right.$ $\left.\mathrm{L}_{\mathrm{S}}\right)$. Here, the symbol $\mathrm{L}_{M}$ denotes the left $\mathrm{MI}$ and symbol $\mathrm{R}_{M}$ represents right $M I$ whereas $L_{S}$ and $R_{S}$ represent left and right sides of the computer screen, respectively. The symbol $\cap$ denotes the combination of the two conditions. The class $\left(\mathrm{L}_{\mathrm{M}}\right.$ vs $R_{M}$ ) is a standard situation which considers the total number of trials for each subject, consisting of left and right hand MI with four gaze events for all trials. The $\left(L_{M} \cap L_{S}\right)$ vs $\left(R_{M} \cap R_{S}\right)$ is a compatible situation for each subject, and considers the total number of trials when left MI appears on left side of computer screen and right $\mathrm{MI}$ appears on right side of computer screen. $\left(L_{M} \cap R_{S}\right)$ vs $\left(R_{M} \cap L_{S}\right)$ is an incompatible situation for each subject wherein we considered only the situation when left MI appears on right side of computer screen and right MI appears on left side of computer screen for all trials.

Tables II, III, and IV present the accuracies of MI (EEG only) (in \%) and Table V provide the ITR (hybrid BCI) in bps. The accuracies are obtained with motor imagery with two classes, $\mathrm{N}_{\text {out }}=2$ (left and right). In the Table II, the achievable mean accuracy of standard condition for all
TABLE I. Gaze detection accuracy

\begin{tabular}{|c|c|c|c|c|c|l|}
\hline \hline Sub & \multicolumn{5}{|c|}{ Accuracy (\%) } & $\begin{array}{l}\text { Level of } \\
\text { expertise }\end{array}$ \\
\hline & Total & 1 & 2 & 3 & 4 & \\
\hline \hline S01 & 100 & 100 & 100 & 100 & 100 & Moderate \\
S02 & 97.5 & 100 & 95 & 100 & 93 & Novice \\
S03 & 99.1 & 100 & 100 & 96 & 100 & Experienced \\
S04 & 91.6 & 91 & 100 & 90 & 85.4 & Moderate \\
S05 & 100 & 100 & 100 & 100 & 100 & Experienced \\
S06 & 95.9 & 100 & 100 & 100 & 83.3 & Novice \\
S07 & 82.5 & 82 & 100 & 75 & 73 & Novice \\
\hline Mean & $\mathbf{9 5 . 2}$ & $\mathbf{9 6 . 1}$ & $\mathbf{9 9 . 3}$ & $\mathbf{9 4 . 4}$ & $\mathbf{9 0 . 7}$ & \\
Std. & $\mathbf{6 . 3}$ & $\mathbf{7 . 1}$ & $\mathbf{1 . 9}$ & $\mathbf{9 . 3}$ & $\mathbf{1 0 . 5}$ & \\
\hline \hline
\end{tabular}

TABLE II. Single trial detection performance for $\left(\mathrm{L}_{\mathrm{M}}\right)$ Vs $\left(\mathrm{R}_{\mathrm{M}}\right)$ in standard state.

\begin{tabular}{|c|cccc|cc|c|}
\hline \hline \multirow{2}{*}{ Sub } & \multicolumn{4}{|c|}{ SVM } & \multicolumn{2}{c|}{ kNN } & \multirow{2}{*}{ LDA } \\
\cline { 2 - 7 } & Linear & Quad. & Poly. & Gauss. & K=1 & K=5 & \\
\hline \hline S01 & 85.0 & 77.5 & 77.5 & 82.5 & 72.5 & 80.0 & 85.0 \\
S02 & 62.5 & 50.0 & 50.0 & 52.5 & 65.0 & 55.0 & 62.5 \\
S03 & 57.5 & 55.0 & 55.0 & 55.0 & 50.0 & 47.5 & 55.0 \\
S04 & 55.0 & 50.0 & 55.0 & 55.0 & 62.5 & 60.0 & 52.5 \\
S05 & 55.0 & 52.5 & 50.0 & 47.5 & 47.5 & 57.5 & 47.0 \\
S06 & 57.5 & 60.0 & 50.0 & 55.0 & 60.0 & 52.5 & 55.0 \\
S07 & 60.0 & 57.0 & 70.0 & 60.0 & 62.5 & 65.0 & 60.0 \\
\hline Mean & $\mathbf{6 1 . 8}$ & $\mathbf{5 7 . 4}$ & $\mathbf{5 8 . 2}$ & $\mathbf{5 8 . 2}$ & $\mathbf{6 0 . 0}$ & $\mathbf{5 9 . 6}$ & $\mathbf{5 9 . 6}$ \\
Std. & $\mathbf{1 0 . 6}$ & $\mathbf{9 . 6}$ & $\mathbf{1 1 . 1}$ & $\mathbf{1 1 . 3}$ & $\mathbf{8 . 7}$ & $\mathbf{1 0 . 6}$ & $\mathbf{1 2 . 3}$ \\
\hline \hline
\end{tabular}

TABLE III. Single trial detection performance for $\left(\mathrm{L}_{M} \cap \mathrm{Ls}\right) \mathrm{Vs}\left(\mathrm{R}_{\mathrm{M}} \cap\right.$ Rs) in compatible state.

\begin{tabular}{|c|cccc|cc|c|}
\hline \hline \multirow{2}{*}{ Sub } & \multicolumn{4}{|c|}{ SVM } & \multicolumn{2}{|c|}{ kNN } & \multirow{2}{*}{ LDA } \\
\cline { 2 - 7 } & Linear & Quad. & Poly. & Gauss. & K=1 & K=5 & \\
\hline \hline S01 & 90.0 & 90.0 & 80.0 & 90.0 & 85.0 & 80.0 & 85.0 \\
S02 & 55.0 & 55.0 & 40.0 & 55.0 & 70.0 & 45.0 & 65.0 \\
S03 & 65.0 & 55.0 & 55.0 & 55.0 & 60.0 & 55.0 & 50.0 \\
S04 & 54.3 & 56.7 & 61.7 & 55.0 & 61.7 & 66.7 & 53.3 \\
S05 & 65.0 & 60.0 & 60.0 & 65.0 & 50.0 & 55.0 & 65.0 \\
S06 & 65.0 & 60.0 & 50.0 & 60.0 & 70.0 & 65.0 & 65.0 \\
S07 & 58.3 & 60.0 & 71.6 & 68.3 & 58.5 & 58.3 & 66.6 \\
\hline Mean & $\mathbf{6 4 . 7}$ & $\mathbf{6 2 . 4}$ & $\mathbf{6 2 . 3}$ & $\mathbf{6 4 . 1}$ & $\mathbf{6 5 . 0}$ & $\mathbf{6 0 . 7}$ & $\mathbf{6 4 . 3}$ \\
Std. & $\mathbf{1 2 . 1}$ & $\mathbf{1 2 . 4}$ & $\mathbf{1 3 . 3}$ & $\mathbf{1 2 . 6}$ & $\mathbf{1 1 . 2}$ & $\mathbf{1 1 . 1}$ & $\mathbf{1 1 . 3}$ \\
\hline \hline
\end{tabular}

TABLE IV. Single trial detection performance for $\left(L_{M} \cap R_{s}\right)$ Vs $\left(R_{M} \cap\right.$ $\mathrm{L}_{\mathrm{s}}$ ) in incompatible state.

\begin{tabular}{|c|cccc|cc|c|}
\hline \hline \multirow{2}{*}{ Sub } & \multicolumn{4}{|c|}{ SVM } & \multicolumn{2}{c|}{ kNN } & \multirow{2}{*}{ LDA } \\
\cline { 2 - 7 } & Linear & Quad. & Poly. & Gauss. & K=1 & K=5 & \\
\hline \hline S01 & 85.0 & 63.3 & 61.7 & 80.0 & 65.0 & 66.7 & 78.3 \\
S02 & 60.0 & 45.0 & 50.0 & 50.0 & 40.0 & 45.0 & 60.0 \\
S03 & 56.6 & 51.7 & 56.7 & 48.3 & 43.3 & 46.0 & 53.3 \\
S04 & 55.0 & 40.0 & 45.0 & 40.0 & 60.0 & 45.0 & 50.0 \\
S05 & 59.1 & 58.0 & 55.0 & 58.3 & 48.3 & 50.0 & 54.5 \\
S06 & 61.0 & 60.0 & 50.0 & 58.3 & 60.0 & 60.0 & 56.6 \\
S07 & 55.0 & 55.0 & 65.0 & 60.0 & 65.0 & 55.0 & 60.0 \\
\hline Mean & $\mathbf{6 1 . 7}$ & $\mathbf{5 3 . 3}$ & $\mathbf{5 4 . 8}$ & $\mathbf{5 6 . 4}$ & $\mathbf{5 4 . 5}$ & $\mathbf{5 2 . 5}$ & $\mathbf{5 9 . 0}$ \\
Std. & $\mathbf{1 0 . 6}$ & $\mathbf{8 . 4}$ & $\mathbf{7 . 0}$ & $\mathbf{1 2 . 6}$ & $\mathbf{1 0 . 5}$ & $\mathbf{8 . 4}$ & $\mathbf{9 . 3}$ \\
\hline \hline
\end{tabular}

subjects is $61.8 \pm 10.6 \%$ computed by SVM linear kernel whereas the mean accuracy of $\left(L_{M} \cap L_{S}\right)$ vs $\left(R_{M} \cap R_{S}\right)$ and $\left(\mathrm{L}_{\mathrm{M}} \cap \mathrm{R}_{\mathrm{S}}\right)$ vs $\left(\mathrm{R}_{\mathrm{M}} \cap \mathrm{L}_{\mathrm{S}}\right)$ conditions are $64.7 \pm 12.1 \%$ and $61.7 \pm 10.6 \%$ achieved, respectively with SVM linear kernel, shown in the Table III and IV. The highest mean accuracy was achieved in compatible condition using SVM linear kernel. However, the gain in performance achieved under the compatible condition is not statistically significant with the small number of novice subject group used in the trials.

In addition, in the Table $\mathrm{V}$, we provide the ITR using linear SVM method for the combination of gaze and EEG when the system is used for 4 and 8 commands, by combining the selected item, and MI detection for all three conditions. The proposed hybrid BCI achieved the highest 
TABLE V. ITR in bps by SVM linear for MI (EEG only $\left(\mathrm{N}_{\text {out }}=2\right)$ ), gaze (eye tracker only $\left(\mathrm{N}_{\text {out }}=4\right)$ ), and hybrid BCI $\left(\mathrm{N}_{\text {out }}=8\right)$ for all conditions.

\begin{tabular}{|c|ccc|ccc|ccc|}
\hline \hline Sub & \multicolumn{3}{|c|}{$\left(\mathbf{L}_{\mathbf{M}}\right) \mathbf{V s}\left(\mathbf{R}_{\mathbf{M}}\right)$} & \multicolumn{2}{c|}{$\begin{array}{c}\left(\mathbf{L}_{\mathbf{M}} \cap \mathbf{L s}\right) \mathbf{V s}\left(\mathbf{R}_{\mathbf{M}}\right. \\
\cap \mathbf{R s})\end{array}$} & \multicolumn{2}{c|}{$\begin{array}{c}\left(\mathbf{L}_{\mathbf{M}} \cap \mathbf{R}_{\mathbf{s}}\right) \mathbf{V s}\left(\mathbf{R}_{\mathbf{M}}\right. \\
\left.\cap \mathbf{L}_{\mathbf{s}}\right)\end{array}$} \\
\hline & $\mathbf{2}$ & $\mathbf{4}$ & $\mathbf{8}$ & $\mathbf{2}$ & $\mathbf{4}$ & $\mathbf{8}$ & $\mathbf{2}$ & $\mathbf{4}$ & $\mathbf{8}$ \\
\hline \hline S01 & 0.4 & 2.0 & 2.0 & 0.5 & 2.0 & 2.3 & 0.4 & 2.0 & 2.0 \\
$\mathbf{S 0 2}$ & 0.1 & 1.8 & 0.9 & 0.0 & 1.8 & 0.7 & 0.0 & 1.8 & 0.9 \\
$\mathbf{S 0 3}$ & 0.0 & 1.9 & 0.8 & 0.1 & 1.9 & 1.1 & 0.0 & 1.9 & 0.8 \\
$\mathbf{S 0 4}$ & 0.0 & 1.5 & 0.6 & 0.0 & 1.5 & 0.6 & 0.0 & 1.5 & 0.6 \\
$\mathbf{S 0 5}$ & 0.0 & 2.0 & 0.7 & 0.1 & 2.0 & 1.1 & 0.0 & 2.0 & 0.9 \\
S06 & 0.0 & 1.7 & 0.8 & 0.1 & 1.7 & 1.0 & 0.0 & 1.7 & 0.9 \\
S07 & 0.0 & 1.1 & 0.6 & 0.0 & 1.1 & 0.5 & 0.0 & 1.1 & 0.5 \\
\hline Mean & $\mathbf{0 . 1}$ & $\mathbf{1 . 7}$ & $\mathbf{0 . 9}$ & $\mathbf{0 . 1}$ & $\mathbf{1 . 7}$ & $\mathbf{1 . 0}$ & $\mathbf{0 . 1}$ & $\mathbf{1 . 7}$ & $\mathbf{0 . 9}$ \\
Std. & $\mathbf{0 . 1}$ & $\mathbf{0 . 3}$ & $\mathbf{0 . 5}$ & $\mathbf{0 . 2}$ & $\mathbf{0 . 3}$ & $\mathbf{0 . 6}$ & $\mathbf{0 . 1}$ & $\mathbf{0 . 3}$ & $\mathbf{0 . 5}$ \\
\hline \hline
\end{tabular}

mean ITR of $1.0 \pm 0.6$ in compatible condition. With the current subset of subjects, only S01 in compatible condition would obtain a gain in performance from combining the BCI and eye tracker to increase the number of commands, as compared to the use of eye tracker only.

\section{DISCUSSION \& CONCLUSION}

In this paper, a hybrid $\mathrm{BCI}$ has been proposed that combines motor imagery detection and eye tracking. Whereas eye-trackers can provide an efficient way to specify the location of an item on a screen, a more user-centered approach needs to be proposed to select the chosen items. In addition, inexpensive eye-trackers are often not reliable in term of the precision of the gaze location, hence limiting the number of items that can be selected at any given time. Selection can be achieved by gazing for a specified duration (i.e. dwell time) on an item, or the detection of an event such as eye-blinking. In this paper, we have investigated the performance that can be obtained with the detection of motor imagery, and how this detection can increase the number of commands. This solution may provide a more convenient alternative communication means especially for differentlyabled people. The eye-tracker can be used for pointing to an item, and BCI can be used as a switch to select the item through brain activity responses. The presented results suggest that the drop of performance is due to the low accuracy of motor imagery detection as only two sensors were used with dry electrodes. Larger number and better quality of electrodes may provide better performance. The use of different modalities may however increase the difficulty in using the system as the user has to pay attention to the location of his gaze and to the movement imagery. Furthermore, naïve subjects have to get used to both the eyetracker, and the motor imagery task [18],[19].

The performances have been evaluated for three different classification scenarios corresponding to the effect of the similarity between the gaze direction (left/right) and the MI task (left/right) (standard, compatible, and incompatible). The results indicated a higher accuracy for the detection of commands with the same orientation between gaze and motor imagination. Thus, this effect should be taken into account while designing gaze-MI based BCI paradigms. Specifically, the experimental design could incorporate incompatible condition if the emphasis is on having a larger number of choices despite a slight dip in accuracy. This type of paradigms has been widely applied in cognitive psychology and neuroscience to study various cognitive abilities, e.g. the Simon effect [20]. They also used this paradigm for the diagnosis and characterization of psychiatric and neurological disorders [21],[22]. Further works will extend the proposed approach with optimized parameters to increase motor imagery detection.

\section{REFERENCES}

[1] G. Prasad, et al., "Applying a brain-computer interface to support motor imagery practice in people with stroke for upper limb recovery: a feasibility study.,"J. Neuroeng. Rehabil., vol. 7, no. 1, pp. 1-17, Jan. 2010.

[2] a. Nijholt and D. Tan, "Brain-Computer Interfacing for Intelligent Systems,” IEEE Intell. Syst., vol. 23, no. 3, pp. 72-79, May 2008.

[3] V. Kaiser, et al., "Investigation of cue-based vertical and horizontal eye movements with electroencephalographic and eye-tracking data.," Clin. Neurophysiol., vol. 120, no. 11, pp. 1988-93, Nov. 2009.

[4] F. Pichiorri, et al., "Sensorimotor rhythm-based brain - computer interface training: the impact on motor cortical responsiveness," $J$. Neural Eng., vol. 8, no. 2, pp. 1-9, 2011.

[5] J. R. Wolpaw, "Assumptions Meet Emerging Realities," J. Mot. Behav., vol. 42, no. 6, pp. 351-353, 2010.

[6] J. R. Wolpaw and D. J. Mcfarland, "Control of a two-dimensional movement signal by a noninvasive brain - computer interface in humans," PNAS, vol. 101, no. 51, 2004.

[7] R. Rupp, et al., "Combining brain - computer interfaces and assistive technologies : state-of-the-art and challenges," Neuroscience, vol. 4, no. 161 , pp. $1-15,2010$.

[8] G. Pfurtscheller, et al., "The hybrid BCI.," Front. Neurosci., vol. 4 no. April, p. 30, Jan. 2010.

[9] D. O. Doherty, et al.,"Exploring Gaze-Motor Imagery Hybrid BrainComputer Interface design," in IEEE International Conference on Bioinformatics and Biomedicine, 2014, pp. 335-339.

[10] A. Duchowski, Eye Tracking Methodology, Second. Springer London, 2007.

[11] L. R. Young and D. Sheena, "Methods \& Designs Survey of eye movement recording methods," Behav. Reseach Methods Instrum. vol. 7, no. 5, pp. 397-429, 1975.

[12] X. Yong, M. Fatourechi, R. K. Ward, and G. E. Birch, "The Design of a Point-and-Click System by Integrating a Self-Paced BrainComputer Interface With an Eye-Tracker," IEEE J. Emerg. Sel. Top. Circuits Syst., vol. 1, no. 4, pp. 590-602, Dec. 2011.

[13] S. Amiri, R. Fazel-Rezai, and V. Asadpour, "A Review of Hybrid Brain-Computer Interface Systems," Adv. Human-Computer Interact., vol. 2013, pp. 1-8, 2013.

[14] B. Z. Allison, et al., "Toward a hybrid brain-computer interface based on imagined movement and visual attention.," J. Neural Eng., vol. 7, no. 2, p. 26007, Apr. 2010.

[15] B. H. Kim, M. Kim, and S. Jo, "Quadcopter flight control using a low-cost hybrid interface with EEG-based classification and eye tracking.," Comput. Biol. Med., vol. 51, pp. 82-92, Aug. 2014.

[16] J.-S. Choi, et al., "Enhanced perception of user intention by combining EEG and gaze-tracking for brain-computer interfaces (BCIs).,"Sensors (Basel)., vol. 13, no. 3, pp. 3454-72, Jan. 2013.

[17] C. Shannon and W. Weaver, The mathematical theory of communication. Urbana, IL: University of Illinois Press, 1964.

[18] H. Cecotti, et al.,"Multimodal target detection using single trial evoked EEG responses in single and dual-tasks," in 33nd International IEEE Conference of the Engineering in Medicine and Biology Society, 2011, pp. 6311-6314.

[19] Ryan W. Kasper, et al.,"Isolating the Neural Mechanisms of Interference during Continuous Multisensory Dual-task Performance," J. Cognitive Neuroscience., Vol. 26, No. 3, pp. 476489, March 2015.

[20] B. Hommel, "The Simon effect as tool and heuristic.," Acta Psychol. (Amst)., vol. 136, no. 2, pp. 189-202, Feb. 2011.

[21] C. Beste, et al., "Effects of stimulus-response compatibility on inhibitory processes in Parkinson's disease.," Eur. J. Neurosci., vol. 29, no. 4, pp. 855-60, Feb. 2009.

[22] G.Yong-Liang, et al., "Stimulus-response incompatibility effects on event-related potentials in children with attention-deficit hyperactivity disorder,"Brain Cogn, vol. 43 (1-3), pp. 211-215, 2000. 